\title{
Integrated Proteomic Pipeline Using Multiple Search Engines for Proteogenomic Study with Controlled Protein False Discovery Rate. \\ Supporting Information
}

GunWook Park ${ }^{\#}$, Heeyoun Hwang ${ }^{\#}$, Kwang Hoe Kim, Ju Yeon Lee, Hyun Kyoung Lee, Ji Yeong Park, Eun Sun Ji, Sung-Kyu Robin Park, John R. Yates III, Kyung-Hoon Kwon, Young Mok Park, Hyoung-Joo Lee, Young-Ki Paik, Jin Young Kim*, and Jong Shin Yoo*

${ }^{\#}$ These authors are equally contributed.

*Corresponding authors: jongshin@,kbsi.re.kr and jinyoung@,kbsi.re.kr; Phone: +82 43240 5150; Fax: +82432405159

\section{Content}

Calculation

of

E-values

(Figure

S1)

................................................2

Tandem mass spectra of endogenous peptides of ecotropic viral integration site $2 \mathrm{~A}$ (NX_P22794) protein and their corresponding synthetic peptides. (Figure $\mathrm{S} 2)$ 3

The candidates of 27 missing and 12 uncertain proteins identified from the neXtProt database using the integrated proteomic pipeline (IPP) (Table S1) ..6 
Figure S1. Calculation of E-values from the scores of peptide spectrum matches (PSMs) in search result (SEQUEST, MASCOT, MS-GF+) with the neXtProt database (including reversed protein sequences). The scores from each search result were fitted to a least-squares plot (red solid line) against log-transformed PSMs. E-values were calculated by projecting the target PSMs along the least-squares line in a) CID and b) ETD MS/MS spectra.
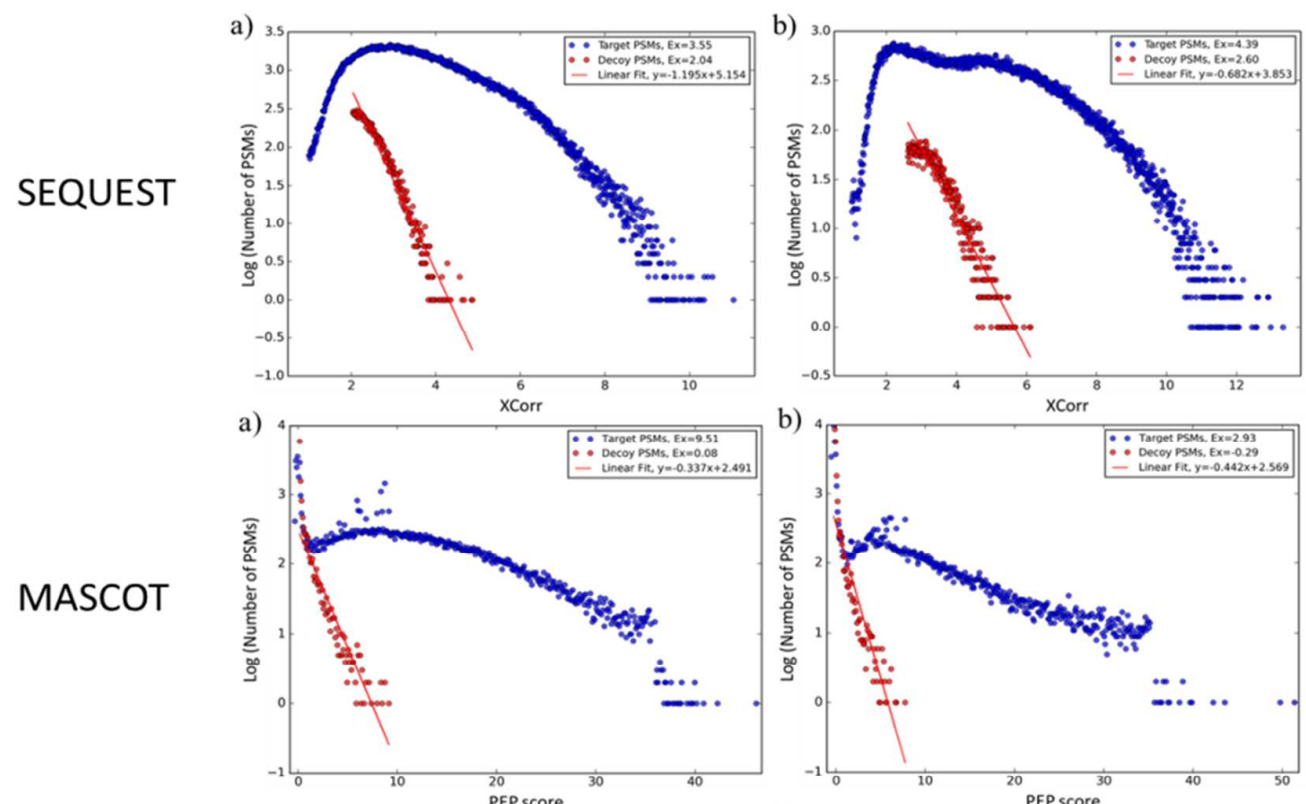

MASCOT
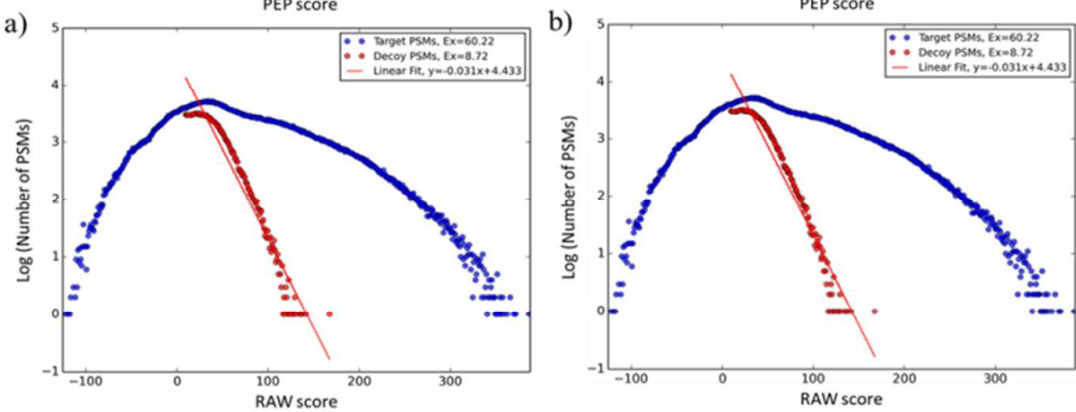
Figure S2. Tandem mass spectra of endogenous peptides of ecotropic viral integration site 2A (NX_P22794) protein and their corresponding synthetic peptides.
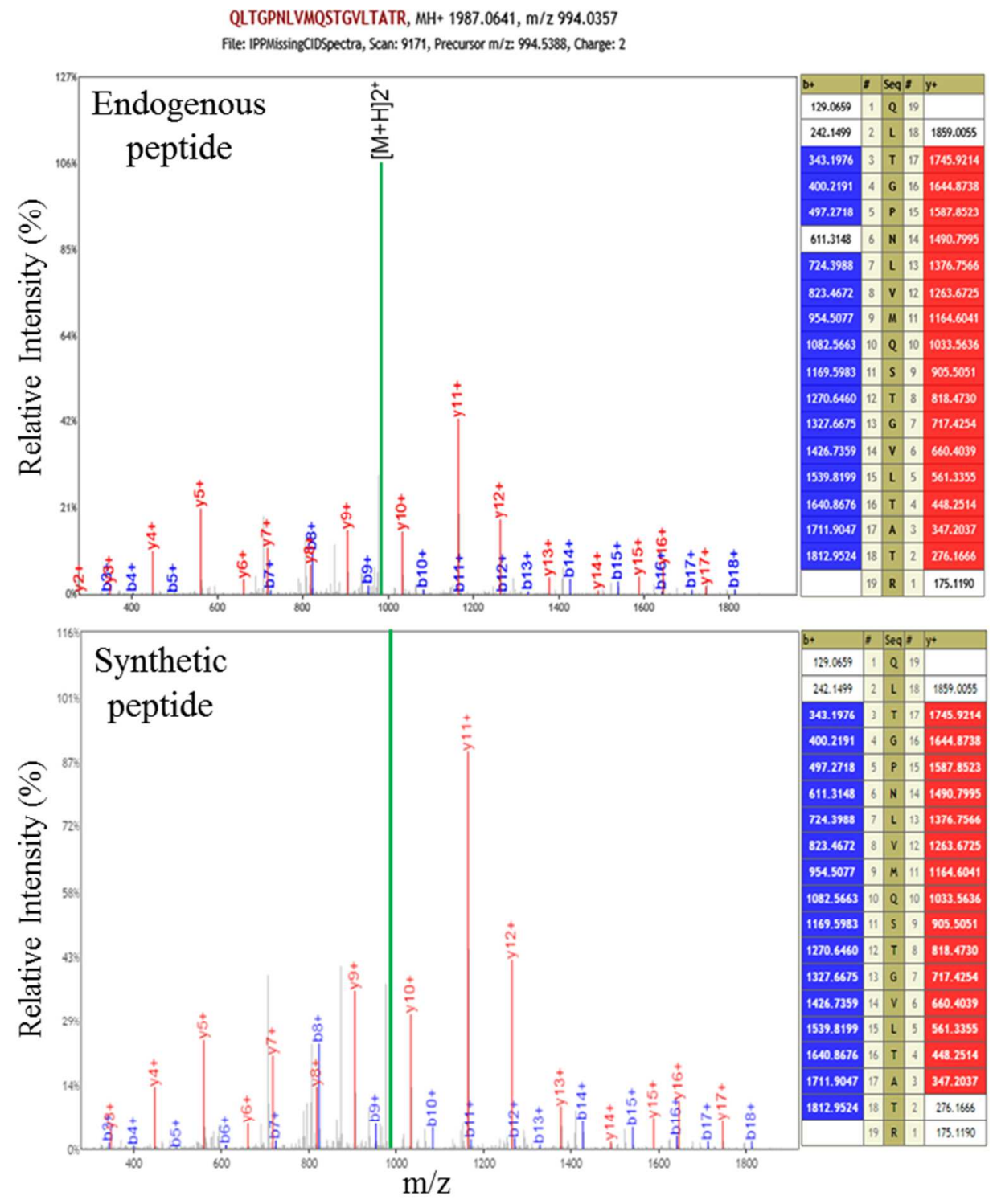


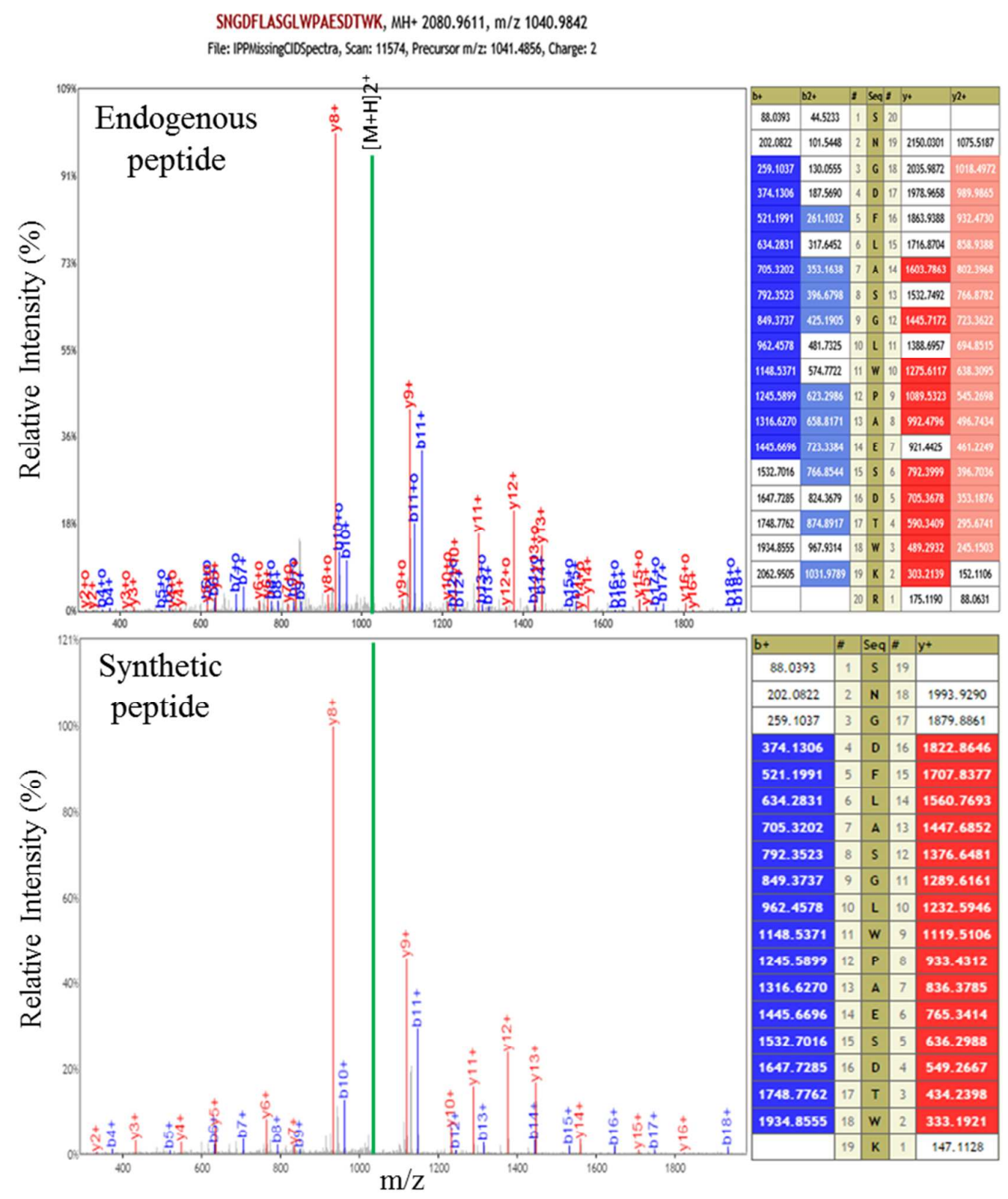




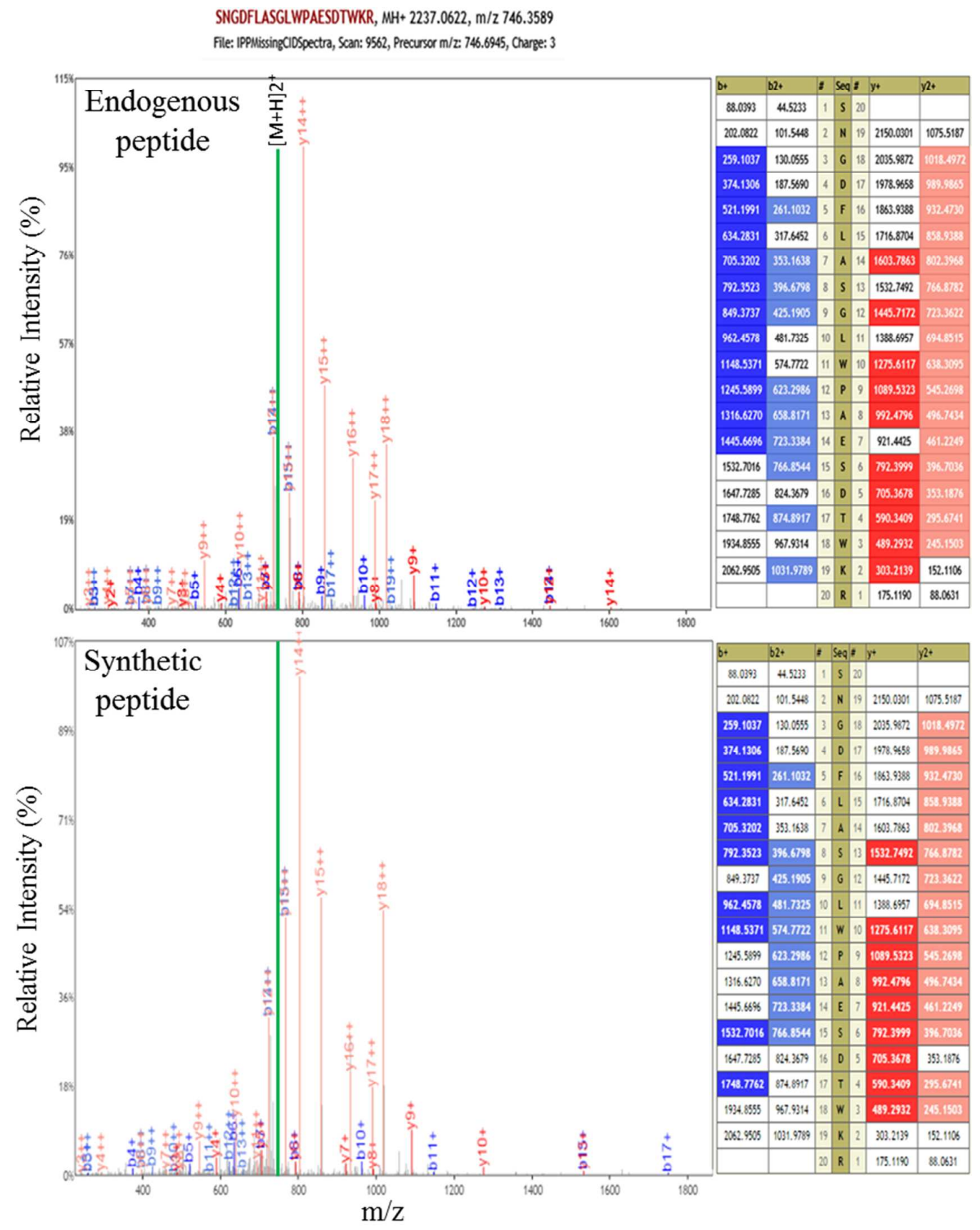


Table S1. The candidates of 27 missing and 12 uncertain proteins identified from the neXtProt database using the integrated proteomic pipeline (IPP) in human hippocampal tissues.

\begin{tabular}{|c|c|c|c|c|c|c|c|c|c|c|c|c|c|c|c|c|c|}
\hline & $\begin{array}{c}\text { Gene } \\
\text { Symbol }\end{array}$ & $\begin{array}{c}\text { neXtProt } \\
\text { Accessions }\end{array}$ & $\begin{array}{l}\text { PE level } \\
\text { (release } \\
09.2014, \\
\text { neXtProt }\end{array}$ & $\begin{array}{c}\text { PE level } \\
\text { (release } \\
02.2016, \\
\text { neXtProt) }\end{array}$ & $\begin{array}{c}\text { Number } \\
\text { of } \\
\text { Unique } \\
\text { Peptide } \\
\text { Sequenc } \\
\text { es } \\
\end{array}$ & $\begin{array}{l}\text { Protein } \\
\text { FDR (\%) }\end{array}$ & $\begin{array}{l}\text { Protei } \\
\text { n E- } \\
\text { score }\end{array}$ & $\begin{array}{l}\text { Protein } \\
\text { Sequence } \\
\text { Coverage } \\
(\%)\end{array}$ & $\begin{array}{l}\text { Chromosomal } \\
\text { position }\end{array}$ & $\begin{array}{c}\text { GPM } \\
\text { DB } \\
\text { (release } \\
09.2014 \text { ) }\end{array}$ & $\begin{array}{c}\text { HPA } \\
\text { (release } \\
11.2014)\end{array}$ & $\begin{array}{l}\text { PeptideAtlas } \\
\text { (release } \\
09.2014 \text { ) }\end{array}$ & $\begin{array}{c}\text { PeptideAtlas } \\
\text { (release } \\
02.2016 \text { ) }\end{array}$ & $\begin{array}{c}\text { Only } \\
\text { SEQUEST } \\
\text { using } \\
\text { neXtProt }\end{array}$ & $\begin{array}{c}\text { Only } \\
\text { MASCOT } \\
\text { using } \\
\text { neXtPto }\end{array}$ & $\begin{array}{c}\text { Only } \\
\text { MS-GF+ } \\
\text { using } \\
\text { neXtProt }\end{array}$ & $\begin{array}{c}\text { Integrated } \\
\text { Proteomic } \\
\text { Pipeline } \\
\text { using } \\
\text { neXtProt }\end{array}$ \\
\hline 1 & AGAP5 & A6NIR3 & PE2 & PE2 & 2 & 0.00 & 5.29 & 16.03 & $10 \mathrm{q} 22.2$ & O & O & (6) & (5) & $x$ & $x$ & $x$ & O \\
\hline 2 & FRMPD3 & Q5JV73 & PE2 & PE1 & 2 & 0.00 & 4.98 & 3.20 & $\mathrm{Xq} 22.3$ & 0 & 0 & (6) & (1) & 0 & 0 & 0 & 0 \\
\hline 3 & LRRTM3 & Q86VH5 & PE2 & PE1 & 2 & 0.00 & 3.41 & 6.04 & $10 \mathrm{q} 21.3$ & $\mathrm{O}$ & $\mathrm{O}$ & (5) & (1) & $\mathrm{O}$ & $\mathrm{O}$ & O & O \\
\hline 4 & PNMA6B & P0C5W0 & PE2 & PE3 & 2 & 0.00 & 3.41 & 9.27 & $\mathrm{Xq} 28$ & $\mathrm{O}$ & $\mathrm{O}$ & (1) & PoCW24 & $x$ & $x$ & $x$ & O \\
\hline 5 & PNMA6C & P0CW26 & PE2 & $\mathrm{Del}^{*}$ & 2 & 0.00 & 3.41 & 8.52 & $\mathrm{Xq} 28$ & $\mathrm{O}$ & $x$ & (7) & (7) & $x$ & $x$ & $x$ & O \\
\hline 6 & CSMD3 & Q7Z407 & PE2 & PE1 & 2 & 0.04 & 2.88 & 1.81 & $8 \mathrm{q} 23.3$ & O & O & $x$ & (1) & 0 & O & 0 & O \\
\hline 7 & EIF4E1B & A6NMX2 & PE2 & PE3 & 2 & 0.04 & 2.77 & 9.09 & $5 \mathrm{q} 35.2$ & $\mathrm{O}$ & $\mathrm{O}$ & (6) & (5) & $x$ & $x$ & $x$ & O \\
\hline 8 & MCF2L2 & Q86YR7 & PE2 & PE2 & 2 & 0.04 & 2.71 & 4.19 & $3 \mathrm{q} 27.1$ & O & O & $x$ & $x$ & $x$ & $x$ & O & O \\
\hline 9 & LRRTM2 & O43300 & PE2 & PE1 & 2 & 0.22 & 2.32 & 6.98 & $5 \mathrm{q} 31.2$ & $\mathrm{O}$ & $\mathrm{O}$ & $x$ & (1) & $\mathrm{O}$ & O & $x$ & $\mathrm{O}$ \\
\hline 10 & EVI2A & P22794 & PE2 & PE2 & 3 & 0.00 & 5.76 & 16.53 & $17 \mathrm{q} 11.2$ & O & O & $x$ & weak & O & O & O & O \\
\hline 11 & CDH9 & Q9ULB4 & PE2 & PE1 & 3 & 0.00 & 3.49 & 6.21 & $5 \mathrm{p} 14.1$ & $\mathrm{x}$ & 0 & (5) & (1) & 0 & 0 & 0 & 0 \\
\hline 12 & SYT10 & Q6XYQ8 & PE2 & PE2 & 3 & 0.00 & 3.40 & 8.99 & $12 \mathrm{p} 11.1$ & $\mathrm{O}$ & $\mathrm{O}$ & $x$ & (2) & $x$ & $x$ & $x$ & O \\
\hline 13 & C18ORF42 & P0CW23 & PE4 & PE1 & 3 & 0.00 & 4.96 & 78.26 & $18 \mathrm{p} 11.31$ & 0 & 0 & $x$ & $x$ & 0 & 0 & 0 & 0 \\
\hline 14 & PCDHGA8 & Q9Y5G5 & PE2 & PE2 & 4 & 0.00 & 5.72 & 5.58 & $5 \mathrm{q} 31.3$ & $\mathrm{O}$ & $\mathrm{O}$ & (7) & (5) & $x$ & $\mathrm{x}$ & $x$ & O \\
\hline 15 & PCDHGA7 & Q9Y5G6 & PE2 & PE2 & 4 & 0.00 & 5.72 & 6.65 & $5 \mathrm{q} 31.3$ & O & O & (7) & (5) & $\mathrm{x}$ & $\mathrm{x}$ & $\mathrm{x}$ & O \\
\hline 16 & $\begin{array}{l}\text { PCDHGA1 } \\
0\end{array}$ & Q9Y5H3 & PE2 & PE2 & 4 & 0.00 & 5.72 & 4.59 & $5 \mathrm{q} 31.3$ & $\mathrm{O}$ & $\mathrm{O}$ & (7) & (5) & $x$ & $x$ & $x$ & O \\
\hline 17 & PCDHGA1 & Q9Y5H4 & PE2 & PE2 & 4 & 0.00 & 5.72 & 5.69 & $5 \mathrm{q} 31.3$ & O & O & (6) & (2) & $x$ & 0 & $\mathrm{x}$ & 0 \\
\hline
\end{tabular}




\begin{tabular}{|c|c|c|c|c|c|c|c|c|c|c|c|c|c|c|c|c|c|}
\hline 18 & GRIK5 & Q16478 & PE2 & PE2 & 4 & 0.00 & 3.80 & 7.96 & $19 \mathrm{q} 13.2$ & 0 & 0 & (4) & (5) & 0 & $x$ & $x$ & $\mathrm{O}$ \\
\hline 19 & SEC14L3 & Q9UDX4 & PE2 & PE1 & 4 & 0.00 & 3.78 & 16.75 & $22 \mathrm{q} 12.2$ & 0 & 0 & (4) & (5) & $x$ & $x$ & $x$ & 0 \\
\hline 20 & CTAGE9 & A4FU28 & PE2 & PE2 & 4 & 0.00 & 3.26 & 7.98 & $6 \mathrm{q} 23.2$ & 0 & $x$ & (4) & (5) & $x$ & $x$ & $x$ & O \\
\hline 21 & RAD21L1 & Q9H4I0 & PE2 & PE2 & 4 & 0.07 & 2.60 & 10.97 & 20p13 & 0 & 0 & (5) & (5) & $x$ & $x$ & $x$ & O \\
\hline 22 & SLC1A6 & P48664 & PE2 & PE2 & 4 & 0.20 & 2.41 & 38.46 & $19 p 13.12$ & $x$ & 0 & (5) & (2) & 0 & $x$ & 0 & 0 \\
\hline 23 & CCDC146 & Q8IYE0 & PE2 & PE1 & 4 & 0.29 & 2.28 & 12.41 & $7 \mathrm{q} 11.23$ & 0 & $x$ & (7) & (5) & $x$ & $x$ & $x$ & $\mathrm{O}$ \\
\hline 24 & $\begin{array}{l}\text { ANKRD30 } \\
\text { B }\end{array}$ & Q9BXX2 & PE2 & PE2 & 4 & 0.70 & 2.18 & 3.74 & $18 \mathrm{p} 11.21$ & 0 & 0 & (5) & (5) & $x$ & $x$ & $x$ & $\mathrm{O}$ \\
\hline 25 & WASH3P & C4AMC7 & PE2 & PE1 & 6 & 0.00 & 5.73 & 15.12 & $15 \mathrm{q} 26.3$ & O & $x$ & (5) & (3) & O & O & O & $\mathrm{O}$ \\
\hline 26 & PCDHGC5 & Q9Y5F6 & PE2 & PE1 & 6 & 0.00 & 5.72 & 11.33 & $5 \mathrm{q} 31.3$ & $x$ & 0 & (5) & (1) & 0 & 0 & 0 & $\mathrm{O}$ \\
\hline 27 & RGPD1 & P0DJD0 & PE2 & PE2 & 7 & 0.00 & 4.57 & 7.09 & $2 \mathrm{p} 11.2$ & 0 & 0 & (5) & $\begin{array}{c}\text { representat } \\
\text { ive }\end{array}$ & $x$ & $x$ & $x$ & 0 \\
\hline 1 & NACAP1 & Q9BZK3 & PE5 & PE5 & 2 & 0.00 & 5.77 & 13.62 & $8 \mathrm{q} 22.3$ & 0 & $x$ & (4) & (1) & O & $x$ & $x$ & 0 \\
\hline 2 & RASA4B & C9J798 & PE5 & PE3 & 2 & 0.00 & 5.74 & 5.60 & $7 \mathrm{q} 22.1$ & 0 & 0 & (5) & (5) & $x$ & 0 & 0 & 0 \\
\hline 3 & TNXA & Q16473 & PE5 & PE5 & 2 & 0.52 & 2.22 & 8.36 & $6 \mathrm{p} 21.33$ & 0 & $x$ & (4) & (2) & $x$ & $x$ & $x$ & O \\
\hline 4 & CES1P1 & Q9UKY3 & PE5 & PE5 & 2 & 0.63 & 2.19 & 11.50 & $16 \mathrm{q} 12.2$ & $x$ & $x$ & (6) & (5) & $x$ & $x$ & $x$ & O \\
\hline 5 & $\begin{array}{l}\mathrm{HSP} 90 \mathrm{AA} \\
4 \mathrm{~Pa}\end{array}$ & Q58FG1 & PE5 & PE5 & 4 & 0.00 & 6.21 & 13.16 & $4 \mathrm{q} 35.2$ & $x$ & $x$ & (4) & (1) & 0 & $x$ & $x$ & O \\
\hline 6 & TUBA4B & Q9H853 & PE5 & PE5 & 6 & 0.00 & 6.27 & 19.50 & $2 \mathrm{q} 35$ & O & $x$ & (6) & (2) & $x$ & O & O & O \\
\hline 7 & PIPSL & $\mathrm{A} 2 \mathrm{~A} 3 \mathrm{~N} 6$ & PE5 & PE5 & 7 & 0.02 & 3.03 & 12.30 & $10 \mathrm{q} 23.33$ & O & $x$ & (1) & (2) & O & 0 & O & O \\
\hline 8 & $\begin{array}{l}\text { UQCRFS1 } \\
\text { P1 }\end{array}$ & P0C7P4 & PE5 & PE5 & 9 & 0.00 & 6.35 & 40.64 & $22 \mathrm{q} 13.1$ & $x$ & $x$ & (6) & (5) & O & $x$ & O & O \\
\hline 9 & ST13P5 & Q8NFI4 & PE5 & PE5 & 10 & 0.00 & 6.35 & 25.20 & $11 \mathrm{p} 15.1$ & O & $x$ & (1) & (1) & O & $x$ & $x$ & O \\
\hline 10 & RAB1C & Q92928 & PE5 & PE5 & 10 & 0.00 & 6.22 & 49.75 & $9 \mathrm{p} 13.2$ & 0 & $x$ & (4) & (2) & $x$ & $x$ & $x$ & O \\
\hline 11 & $\begin{array}{l}\text { HSP90AB4 } \\
\text { P }\end{array}$ & Q58FF6 & PE5 & PE5 & 10 & 0.00 & 6.05 & 16.04 & $15 \mathrm{q} 21.3$ & O & $x$ & (4) & (1) & 0 & $x$ & $x$ & O \\
\hline 12 & POTEKP & Q9BYX7 & PE5 & PE5 & 15 & 0.00 & 6.22 & 31.73 & $2 \mathrm{q} 21.1$ & O & $x$ & (4) & (1) & O & $x$ & $x$ & O \\
\hline
\end{tabular}

*: deleted in latest neXtProt database. 
(1):canonical, (2):marginally distinguished, (3):indistinguishable representative, (4):possibly distinguished, (5):subsumed by, (6):ntt-subsumed by, (7):indistinguishable, (8):insufficient evidence, $X$ :not detected, O:detected 